\title{
Wavelength Shifting Phoswich Detectors for Superior Depth-of-Interaction Resolution
}

\author{
Charles L. Melcher (PI) and Lars Eriksson (Co-PI) \\ GRANT: DE-FG02-08ER64678 \\ PERIOD OF PERFORMANCE: 09/15/2008 - 09/14/2010
}

\section{BACKGROUND}

In order to simultaneously achieve both high spatial resolution and high sensitivity in small Positron Emission Tomography (PET) systems, scintillation detectors must be long in the radial direction as well as able to provide depth-ofinteraction (DOI) information. DOI information is typically provided by constructing detectors from two or more layers of scintillators that are identifiable due to their different decay times. This approach has worked well in tomographs such as the High Resolution Research Tomograph (HRRT, CTI PET Systems, Inc.) in which the emission and excitation bands of the scintillator layers do not overlap each other. However, many potentially important pairs of scintillator crystals exist in which the emission of one crystal is, in fact, absorbed and re-emitted by the second crystal, thus impacting the pulse shape discrimination process used to identify the scintillator layers. These potentially useful pairs of scintillators are unlikely to be implemented in phoswich detectors without a comprehensive understanding of the complex emission that results when one crystal's light is absorbed by the second crystal and then reemitted. Our objective has been to develop a fundamental understanding of the optical phenomena that occur in phoswich detectors and to exploit these phenomena to achieve improved spatial resolution in small high sensitivity PET scanners.

Luminescence phenomena in phoswich detectors include both radioluminescence, i.e. light emission that is excited by gamma rays, and photoluminescence, i.e. the light emission that occurs when light from one crystal is absorbed and re-emitted by the second crystal. Since the shape of the light pulse that ultimately reaches the photosensor is affected by both of these phenomena, a clear understanding their characteristics is important for optimization of the pulse-shape-discrimination process. Our goal is use measured decay times, rise times, emission spectra, and excitation spectra as inputs for simulations of the convolved scintillation light pulse, and to benchmark the simulations to measurements of real phoswich configurations. The results then guide the selection of combinations of scintillators as well as to provide new techniques for extracting the maximum DOI information from thick detectors.

We have assembled and characterized various sets of scintillators that are candidates for high resolution and high sensitivity PET. Some crystals have been grown in our own laboratory while others have been obtained commercially. We are focused on high density and high atomic number compounds that are doped with fast and efficient luminescent activators such as Ce and Pr. We have also investigated the use of wavelength shifters, bandpass filters, and wavelength shifting reflectors in order to optimize the identification of the crystal layers. The scintillators are characterized by means of both radioluminescence and photoluminescence techniques. The time-correlated single photon counting technique is used to measure rise times and decay times under gamma ray excitation as well as under photo excitation with our newly acquired pulsed LED system. The photoluminescence lifetime measurements are crucial to our investigation since radioluminescence and photoluminescence lifetimes are often significantly different due to the effects of energy migration and charge carrier trapping in the radioluminescence mechanism. We paid particular attention to multiple decay components since they can play a significant role in the convolved light pulse in crystal-to-crystal interactions. One post-doc and one Ph.D. student have carried out this research. The results have been reported via oral and poster presentations at international conferences (IEEE NSS-MIC, 2008 and 2009), conference proceedings papers, and papers in Nuclear Instruments and Methods and IEEE Transactions on Nuclear Science.

Our research supports DOE's energy and environmental mission by offering a path to improved high resolution imaging of dynamic biological processes in vivo that is not achievable with conventional imaging technology. In addition, our research contributes directly to the molecular imaging research supported by NIH, particularly in Positron Emission Tomography, by providing the opportunity to image molecular processes with sensitivity and spatial resolution not previously available. Industrial implementation of this technology should be straightforward . 


\section{INTRODUCTION}

The high Resolution Research Tomograph (HRRT) brain scanner is based on phoswich detectors and has excellent spatial resolution characteristics [1]. The present generation HRRT systems [2] use cerium activated $\mathrm{Lu}_{2} \mathrm{SiO}_{5}$ (LSO:Ce) and $\mathrm{Lu}_{0.6} \mathrm{Y}_{1.4} \mathrm{SiO}_{5}$ (LYSO:Ce) scintillators, with detector pixels $2.2 * 2.2 * 10 \mathrm{~mm}^{3}$. LSO:Ce is the front layer and LYSO:Ce is the back layer. The scintillation decay times for the two layers are approximately 40-45 ns for the LSO:Ce and around 55 ns for the LYSO:Ce. The layer selection is based on pulse shape discrimination. Originally $\mathrm{Gd}_{2} \mathrm{SiO}_{5}$ (GSO:Ce) was planned for the back layer of the phoswich but due to cleavage planes in GSO:Ce only a few LSO/GSO detectors were built. Ideally the two components in a phoswich should have the same characteristics, except for a difference in scintillation decay time. The best choice would have been two LSO layers, a fast and a slow version. At the time, however, LYSO was decided to be the best choice.

The new scintillators cerium activated $\mathrm{LuAlO}_{3}(\mathrm{LuAP}: \mathrm{Ce})$ and $(\mathrm{Lu}, \mathrm{Y}) \mathrm{AlO}_{3}(\mathrm{LuYAP}: \mathrm{Ce})$ have excellent characteristics for detection of $511 \mathrm{keV}$ photons thus making them good candidates for detectors in PET. Possibly these scintillators could be used in a phoswich combination, as the back layer with LSO as the front layer. The principal scintillation decay time for both LuAP and LuYAP is short, around $20 \mathrm{~ns}$ and the light output is of the order of one third of LSO. Specifically PET systems with phoswich detectors based on LuYAP and LSO have been suggested [3] and realized [4].

However, the LuYAP:Ce (and the $\mathrm{LaBr}_{3}: \mathrm{Ce}$ ) family of scintillators emits light into the excitation band of LSO:Ce which may complicate the situation. The LuYAP:Ce light will thus excite LSO:Ce resulting in LSO:Ce light with time characteristics of a convolution between LuYAP:Ce and LSO:Ce scintillation times

$$
\langle L u Y A P \rightarrow L S O\rangle(t)=\operatorname{LuYAP}(t) \otimes L S O(t) \quad \text { Eqn. (1) }
$$

From a phoswich point of view this would only be a problem if the scintillation decay time of LuYAP:Ce is shorter than the LSO:Ce scintillation decay time. The shorter LuYAP:Ce decay time will be converted to LSO:Ce decay time and the two components in the phoswich will now have the same decay time and it may turn out to be difficult to identify the two crystals. Looking at the available information of LuYAP:Ce scintillators, however, only a fraction is decaying via the fast channel. Annenkov et al [5] find that LuYAP:Ce with 30\% yttrium has three decay components: $20 \mathrm{ns,} 70 \mathrm{~ns}$ and $400 \mathrm{~ns}$, with branching ratios of $0.5,0.25$ and 0.25 , respectively. Musolino et al [6] find $22 \mathrm{~ns}, 180 \mathrm{~ns}$ and 1170 ns decay times with approximate branching fractions of 0.33 each. This implies that the apparent decay time as seen by the pulse shape discrimination set up is longer than the LSO decay time ( $40 \mathrm{~ns})$ and that a separation between the two crystals is feasible in spite of the LuYAP-LSO interaction.

Green et al [7] looked at phoswich combinations of $\mathrm{LaBr}_{3}: \mathrm{Ce}$ and $\mathrm{LYSO}: \mathrm{Ce}$ and found that the $\mathrm{LaBr}_{3}: \mathrm{Ce}$ crystal could not be identified due to the interaction between the two crystals. The $\mathrm{LaBr}_{3}$ :Ce crystal used in their study was Saint Gobain, BrilLanCe 380 with $5 \%$ Ce with a fast rise time $(<1 \mathrm{~ns})$ and a $\sim 16$ ns principal decay time.

In order to study the interactions between scintillators emitting light in the excitation region of the LSO:Ce family of crystals (LSO:Ce, LYSO:Ce, GSO:Ce and YSO:Ce), we have chosen phoswich combinations of $\mathrm{LaBr}_{3}: \mathrm{Ce} / \mathrm{LSO}_{\mathrm{SO}} \mathrm{Ce}$ and $\mathrm{LaBr}_{3}: \mathrm{Ce}$ /YSO:Ce. Since the $\mathrm{LaBr}_{3}$ light interacts with LSO, a possibility to avoid this interaction is the use of a third scintillator with the same excitation-emission characteristics as LSO but with a decay time sufficiently separated from LSO. If the third scintillator is thin enough, the pulse-shape-discrimination circuit would see this as a LSO decay time signature and the signature of a convolution between $\mathrm{LaBr}_{3}$ and the third scintillator. We have investigated the decoupling of the $\mathrm{LaBr}_{3}: \mathrm{Ce}$ crystal from LSO:Ce by using a thin layer of a separate scintillator that shifts the $\mathrm{LaBr}_{3}$ :Ce light away from the excitation region of LSO:Ce. A thin plastic wavelength shifter (WLS, Eljen EJ299-21, $0.1 \mathrm{~mm}, 3 \mathrm{~ns}$ scintillation decay time) was tried. We also tried a thin YSO:Ce layer $(0.7 \mathrm{~mm}, 60 \mathrm{~ns})$ for this.

\section{METHODS}

\section{A. Experimental set ups}

Two different experimental set ups have been used, a Bollinger-Thomas [8] set up for determination of scintillation time characteristics and a conventional pulse-shape-discrimination set up (PSD). The latter is shown in Fig 1. 


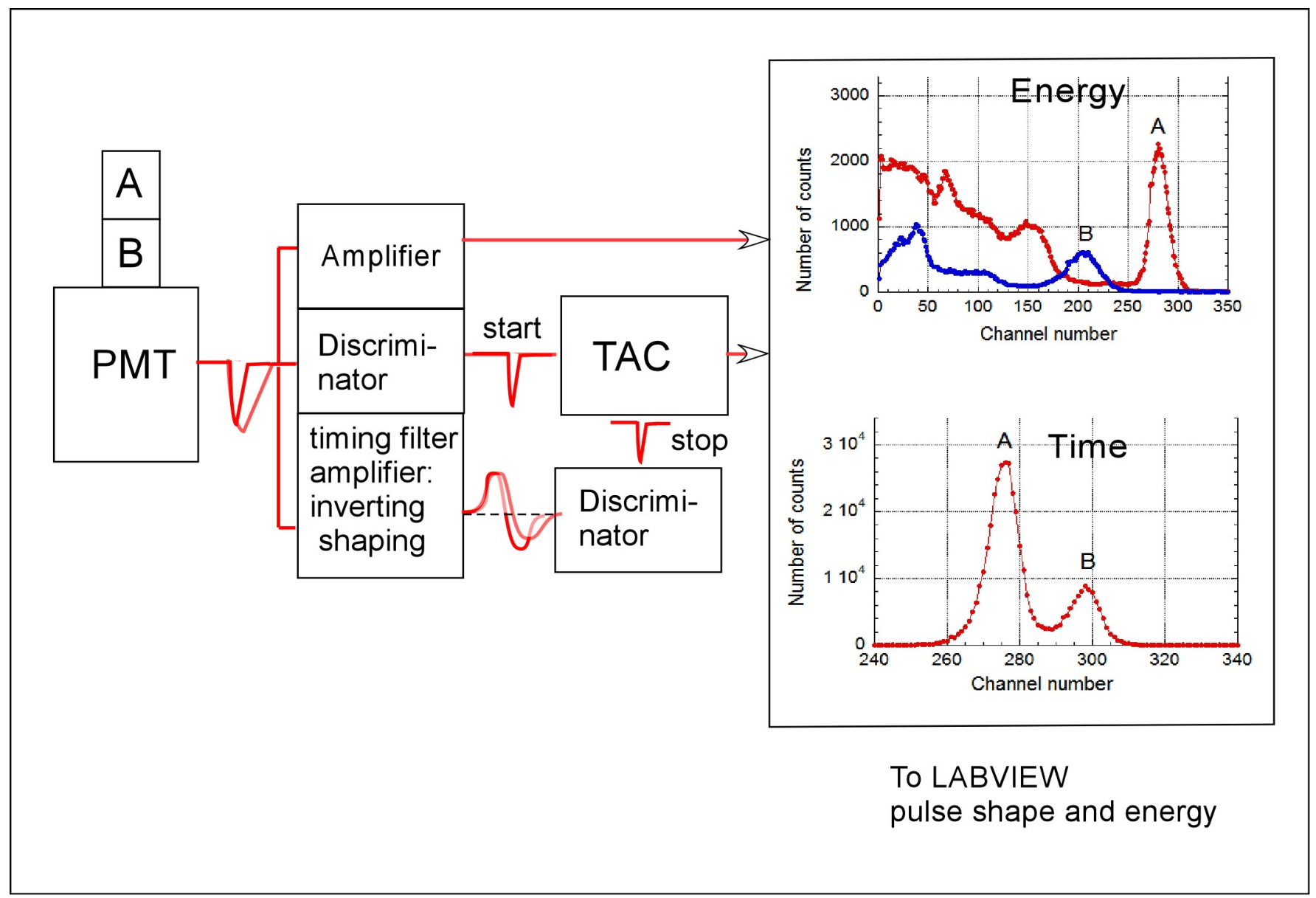

Fig 1. PSD set up. Ideal phoswich situation: A is a fast scintillator and B is a slow scintillator. The phoswich is placed on a photomultiplier (PMT). The PMT signal is directly fed to a fast discriminator to provide a START for a time-amplitude converter (TAC). A STOP signal is provided by a second discriminator after first amplifying, shaping and inverting the PMT signal. A regular amplifier analyzes the PMT signal to provide energy information. A computer with LABVIEW is now used to analyze the pulse shape discrimination signal (time signal) and the energy signals from the two scintillators.

\section{B. The studied phoswich and the wavelength shifter}

Fig 2 shows (left) a phoswich with $\mathrm{LaBr}_{3}: \mathrm{Ce}(0.5 \% \mathrm{Ce}, 25$ ns scintillation decay time $)$ directly coupled to LSO:Ce. The scintillation time characteristics seen by the PMT in this case are the convolved $\mathrm{LaBr}_{3}-\mathrm{LSO}$ pulse and the direct LSO:Ce pulse.

In Fig 2 (right) the commercial wavelength shifter (WLS) from Eljen, is used to shift the $\mathrm{LaBr}_{3}: \mathrm{Ce}$ light away from the LSO:Ce excitation region. The WLS is $0.1 \mathrm{~mm}$ thick and has $>95 \%$ absorption at $375 \mathrm{~nm}$. The emitted wavelength is $430 \mathrm{~nm}$ with a 3 ns scintillation decay time. The scintillation pulse shape in this case is the convolution of $\mathrm{LaBr}_{3}: \mathrm{Ce}$ and the WLS. The resulting convolution is very close to the $\mathrm{LaBr}_{3}$ :Ce pulse shape, but now with an emission wave length of $430 \mathrm{~nm}$, far above the LSO:Ce excitation band. The size of the LSO crystal was $19 * 19^{*} 7.5 \mathrm{~mm}^{3}$. The $\operatorname{LaBr}_{3}:$ Ce was a $1 \mathrm{~cm}$ cube in a cylindrical package with $0.5 \%$ Ce. In a few experiments LSO:Ce was replaced by YSO:Ce of the same dimensions, $19 * 19 * 7.5 \mathrm{~mm}^{3}$. 


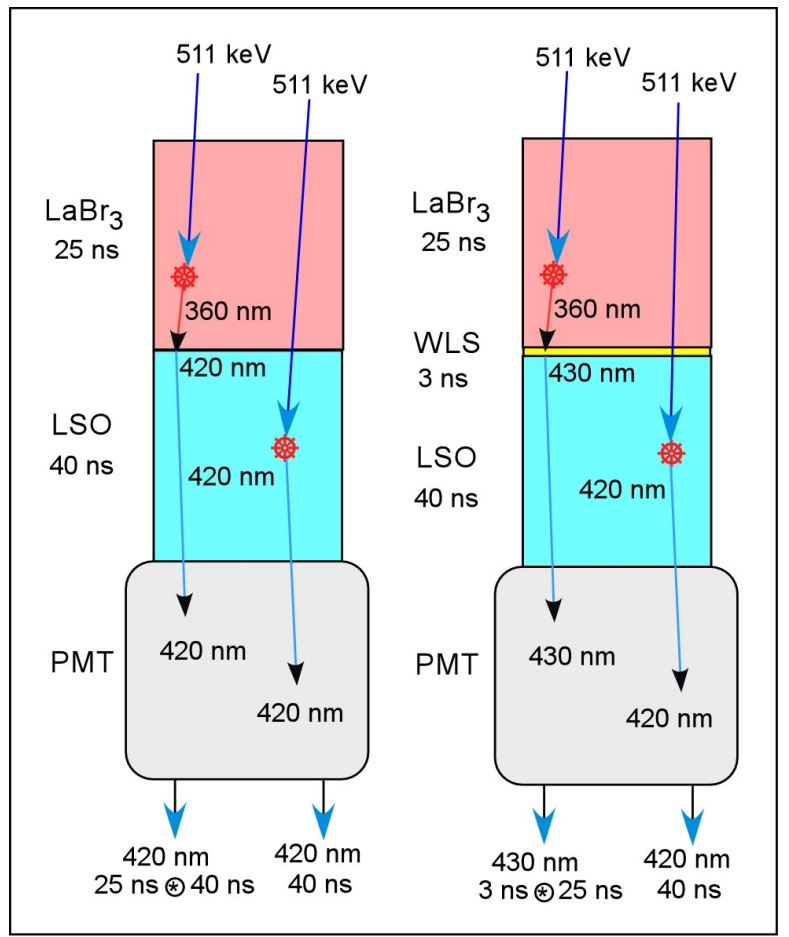

Fig 2. The figures shows the investigated phoswich arrangements, $\mathrm{LaBr}_{3} / \mathrm{LSO}(\mathrm{YSO})$ with and without WLS.

Fig 3 shows the excitation and emission bands of LSO:Ce together with the $\mathrm{LaBr}_{3}$ :Ce emission and the overlap between the LSO:Ce $360 \mathrm{~nm}$ excitation band and the $\mathrm{LaBr}_{3}:$ Ce emission band. The absorption band of the WLS around $360 \mathrm{~nm}$ is also shown.

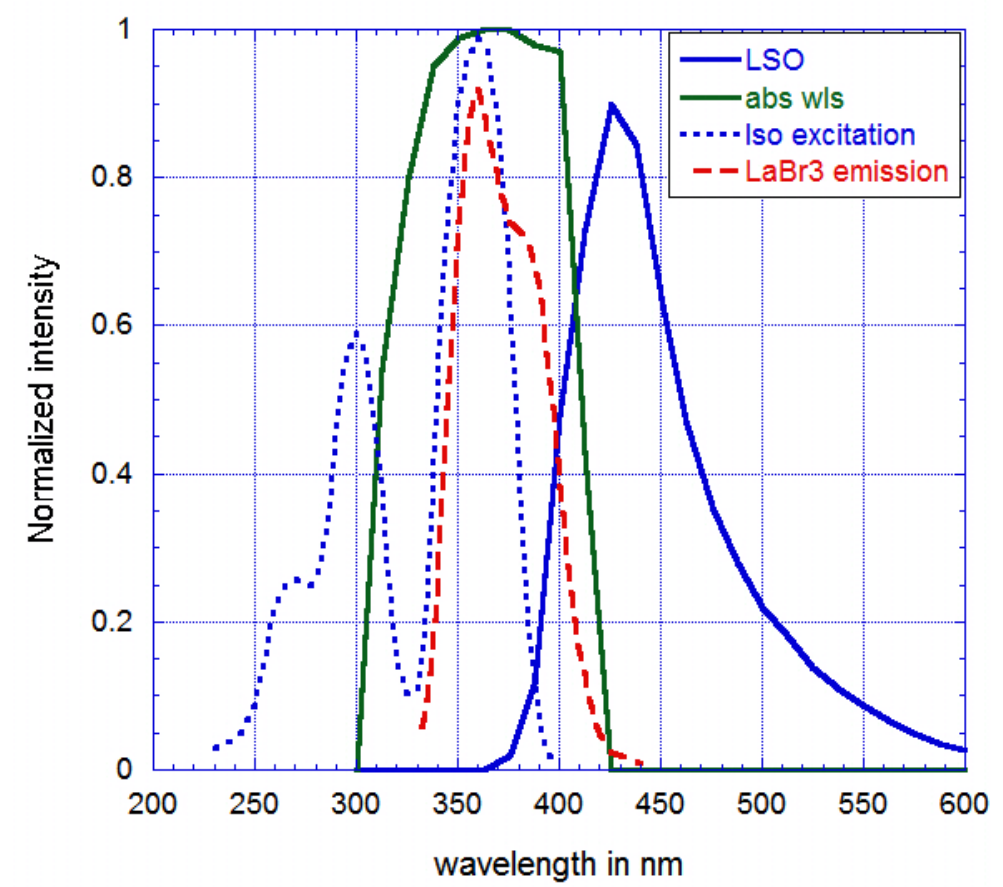

Fig 3. LSO excitation (dotted blue) and emission (blue) bands, the $\mathrm{LaBr}_{3}$ emission (dotted red) and WLS absorption band (green). The WLS emits at 430 $\mathrm{nm}$ (not shown).

C. Scintillation decay time measurements 
Fig 4 shows the initial Bollinger-Thomas experiments with a $\mathrm{LaBr}_{3} / \mathrm{LSO}$ and a $\mathrm{LaBr}_{3} / \mathrm{YSO}$ phoswich in order to determine the interaction between $\mathrm{LaBr}_{3}$ :Ce scintillation decay and the LSO:Ce and YSO:Ce scintillation decay. The $\mathrm{LaBr}_{3}: \mathrm{Ce}$ scintillator was placed on the YSO:Ce or the LSO:Ce crystal in such a way that neither of the two PMTs used for the experiment could directly see $\mathrm{LaBr}_{3}$ light. Events in the $\mathrm{LaBr}_{3}: \mathrm{Ce}$ crystal could only be observed via its light entering the YSO:Ce or the LSO:Ce scintillator.

The experiment was made in two parts. In the first part a radioactive source $\left({ }^{241} \mathrm{Am}\right.$ or $\left.{ }^{137} \mathrm{Cs}\right)$ was placed on the $\mathrm{LaBr}$ : $\mathrm{Ce}$ scintillator, directly coupled to the YSO:Ce or the LSO:Ce crystal. The acquisition was run for one hour. For the second part a light blocker (black tape) was placed between the two scintillators to prevent $\mathrm{LaBr}_{3}$ light from entering the YSO (LSO) crystal. This acquisition was also run for an hour. The reason was to find the fraction of radiation that passed through the $\mathrm{LaBr}_{3}: \mathrm{Ce}$ crystal and interacted with the YSO:Ce or the LSO:Ce crystal. By subtracting the two measurements the convolved $\mathrm{LaBr}_{3} / \mathrm{YSO}$ (or $\mathrm{LaBr}_{3} / \mathrm{LSO}$ ) information could be determined. Fig 5 shows the results for $\mathrm{LaBr}_{3}: \mathrm{Ce}$ light convolved with YSO:Ce activated with a ${ }^{241} \mathrm{Am}$ source and Fig 6 shows the results for $\mathrm{LaBr}_{3}: \mathrm{Ce}$ light convolved with LSO:Ce and using a ${ }^{137} \mathrm{Cs}$ source. The data fit is based on the function

$Y(M 0)=m 5+m 1 *\left(e^{-(M 0-c h 0) / m 3}-e^{-(M 0-c h 0) / m 2}\right) \operatorname{Eqn}(2)$

where $\mathrm{Y}(\mathrm{M} 0)$ is the scintillation decay data as a function of the scintillation decay time, m5 is the background, m3 is the scintillation decay time and $\mathrm{m} 2$ is the scintillation rise time. In fig. 5 the background is set to 1 . Channel ch0 is the initial time channel of the fit.
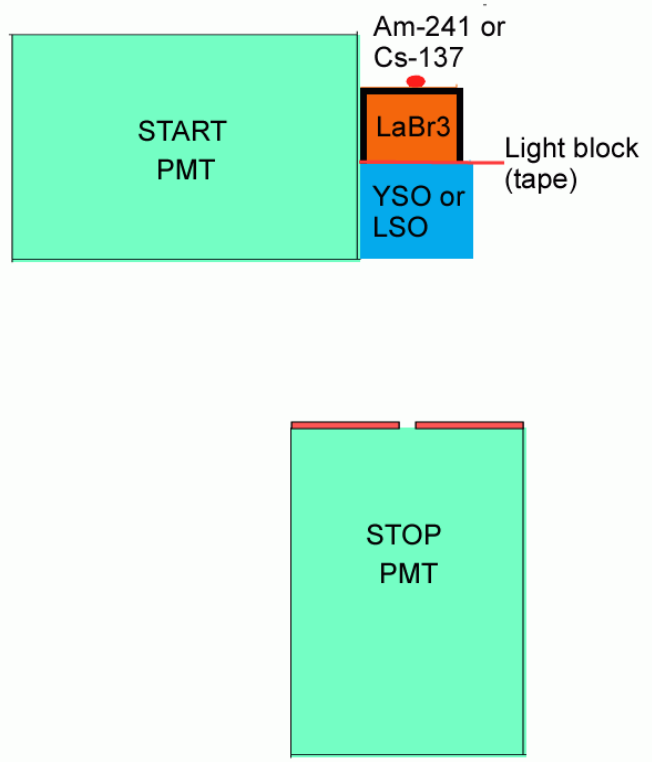

Fig 4. The Bollinger-Thomas set up showing the phoswich configuration. 


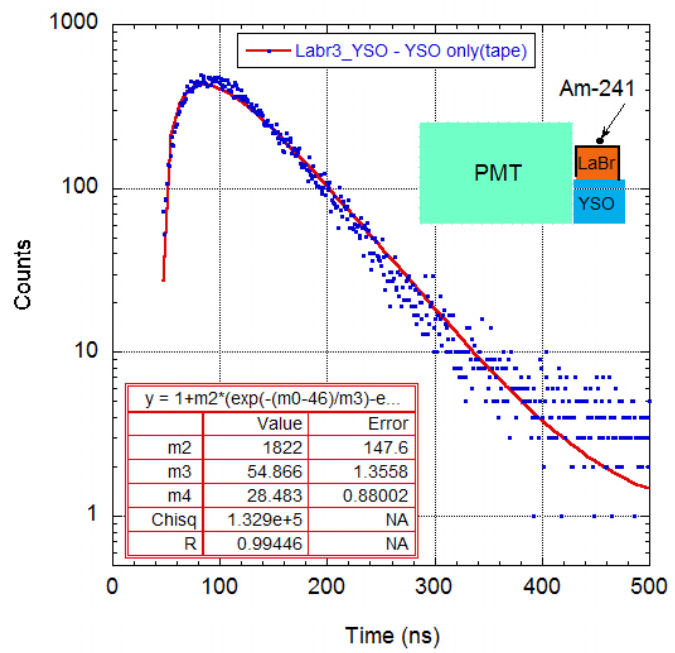

Fig 5. Convolution data for the $\mathrm{LaBr}_{3} / \mathrm{YSO}$ phoswich combination as determined in the Bollinger-Thomas set up with a ${ }^{241}$ Am source. The individual decay times for the two scintillators are $25 \mathrm{~ns}$ for the $\mathrm{LaBr}_{3}: \mathrm{Ce}$ crystal and $60 \mathrm{~ns}$ for the YSO:Ce crystal.

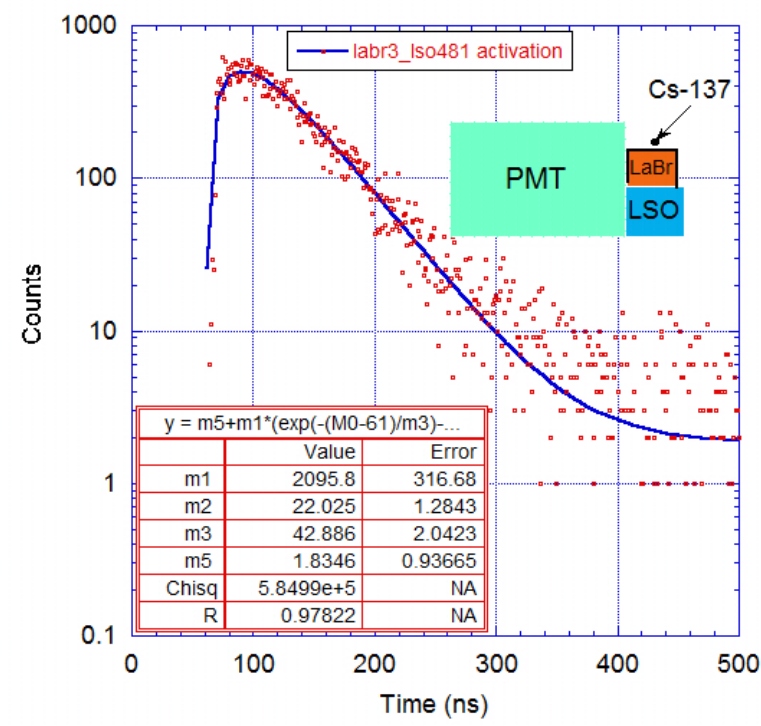

Fig 6. Convolution data for the $\mathrm{LaBr}_{3} / \mathrm{LSO}$ phoswich combination as determined in the Bollinger-Thomas set up with a ${ }^{137} \mathrm{Cs}$ source. The individual decay times for the two scintillators are $25 \mathrm{~ns}$ for the $\mathrm{LaBr}_{3}: \mathrm{Ce}$ crystal and $43 \mathrm{~ns}$ for the LSO:Ce crystal.

\section{Pulse shape discrimination measurements}

This part of the investigation consisted of $\mathrm{LaBr}_{3} / \mathrm{YSO}$ and $\mathrm{LaBr}_{3} / \mathrm{LSO}$ phoswich data with and without the WLS, and $\mathrm{LaBr}_{3} / \mathrm{LSO}$ data with a thin YSO:Ce crystal as a wavelength shifter. All data were acquired with the set-up shown in Fig 1. The results are shown as Figures 7-11. The time scale of the PSD time spectra in Figures 7-11 is around $0.5 \mathrm{~ns}$ per channel.

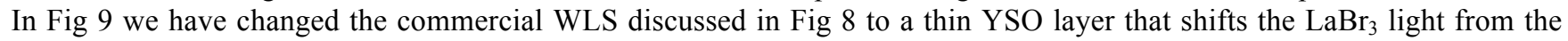
LSO excitation band. The $\mathrm{LaBr}_{3}$ signal now has largely YSO timing characteristics.

In Fig 10 and 11 we show the PSD results for a $\mathrm{LaBr}_{3} / \mathrm{LSO}$ phoswich with and without a wavelength shifter in between.

When placing the $\mathrm{LaBr}_{3}$ crystal directly on the LSO scintillator in order to inspect the PSD timing response (see Fig 11), it was surprisingly found that there were two time peaks when only one was expected.

The peak at channel 415 was the LSO peak as seen from Fig 10a. In addition we found a time peak representing a longer decay time. By inspecting the energy spectra gated by the two peaks we found that the second peak truly represented the LaBr 3 scintillator. 


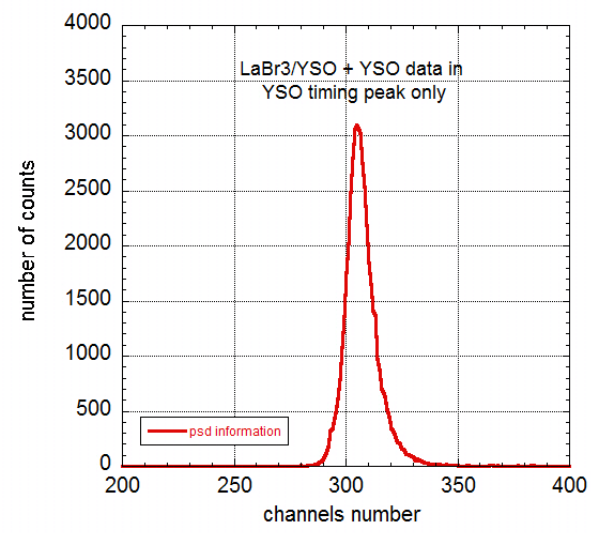

Fig. 7a. $\mathrm{LaBr}_{3} / \mathrm{YSO}$ PSD timing and

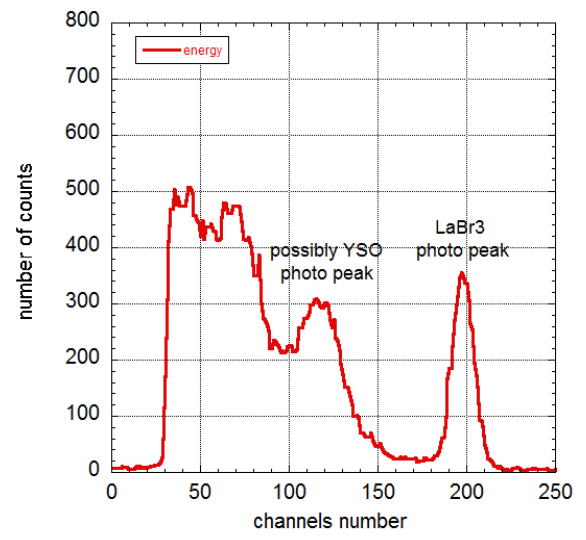

Fig 7 b. $\mathrm{LaBr}_{3} / \mathrm{YSO}$ energy data.

$\mathrm{The}_{\mathrm{LaBr}}$ : $\mathrm{Ce}$ crystal interacts with the YSO:Ce scintillator entering the PMT as largely YSO:Ce timing information The energy information from the two scintillators is thus mixed and can not be separated.

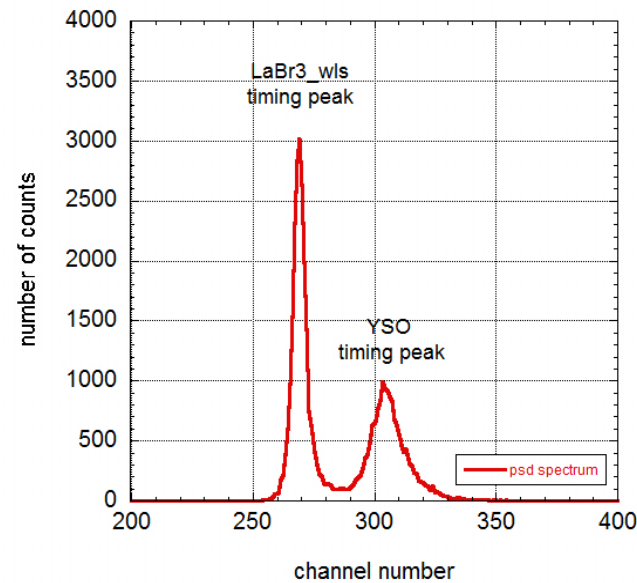

Fig $8 \mathrm{a}$

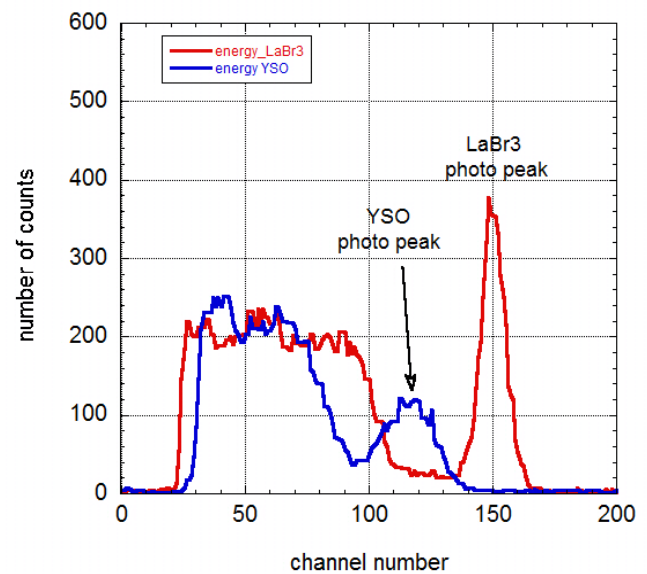

Fig $8 b$

The PSD timing spectra for a $\mathrm{LaBr}_{3}$ YYSO phoswich with WLS in between. The WLS shifts the $\mathrm{LaBr}_{3}$ emission from the YSO excitation band and no interactions takes place between the $\mathrm{LaBr}_{3}$ and the YSO. The $\mathrm{LaBr}_{3}$ and the YSO time peaks are well separated as seen in Fig 8a. In Fig 8b the PSD energy spectra for a $\mathrm{LaBr}_{3} / \mathrm{YSO}$ phoswich with a WLS in between is shown. The energy information from the $\mathrm{LaBr}_{3}$ and the YSO crystals are well separated and can be simultaneously acquired.

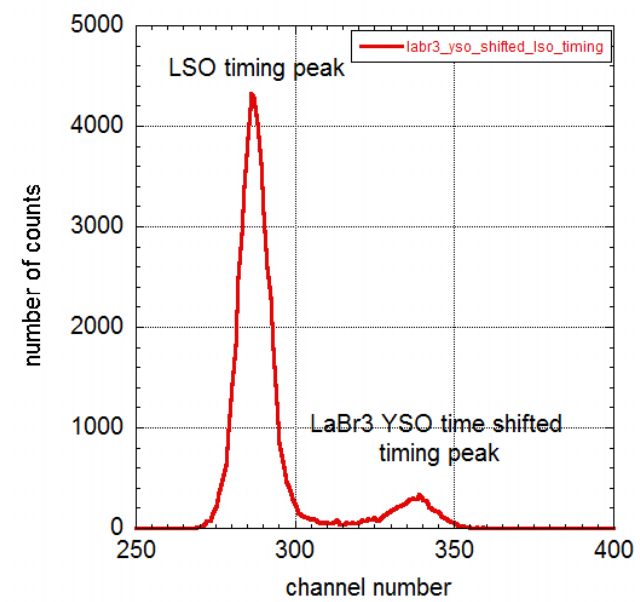

Fig 9 a

In Fig 9 the PSD results of a $\mathrm{LaBr}_{3} / \mathrm{LSO}$ phoswich using a thin YSO:Ce layer in between as a wavelength shifter is shown. The timing spectra shows a good separation and Fig 9b. shows that the resulting energy spectra from the $\mathrm{LaBr}_{3}$ and the $\mathrm{LSO}$ crystals can be acquired simultaneously.

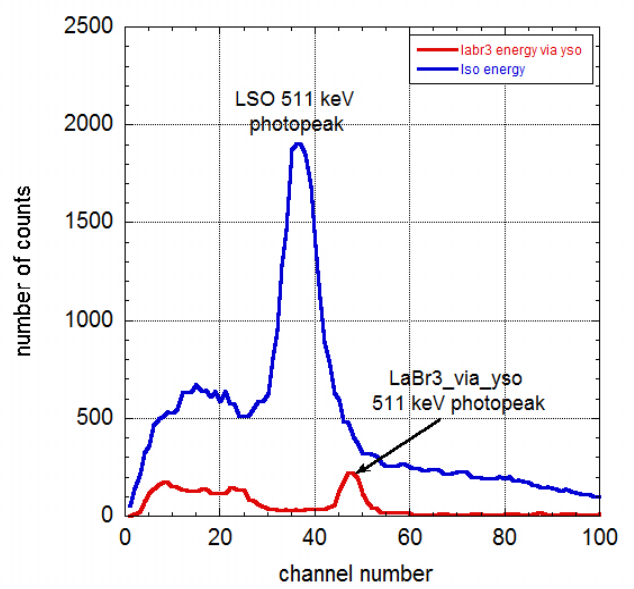

Fig 9b 
Final Technical Report

DE-FG02-08ER64678

$09 / 15 / 2008-09 / 14 / 2010$

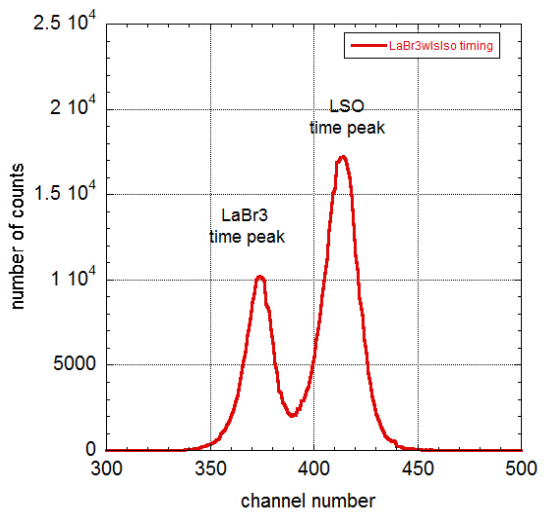

Fig 10a. PSD information for a $\mathrm{LaBr}_{3} / \mathrm{LSO}$ phoswich, with the WLS in between. Timing information

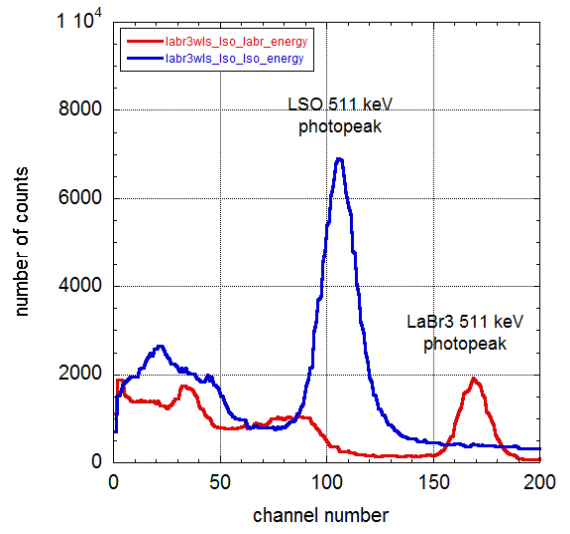

Fig 10b. PSD information for a $\mathrm{LaBr}_{3} / \mathrm{LSO}$ phoswich, with the WLS in between. The two crystals can be easily identified and the two energy spectra can be acquired simultaneously.

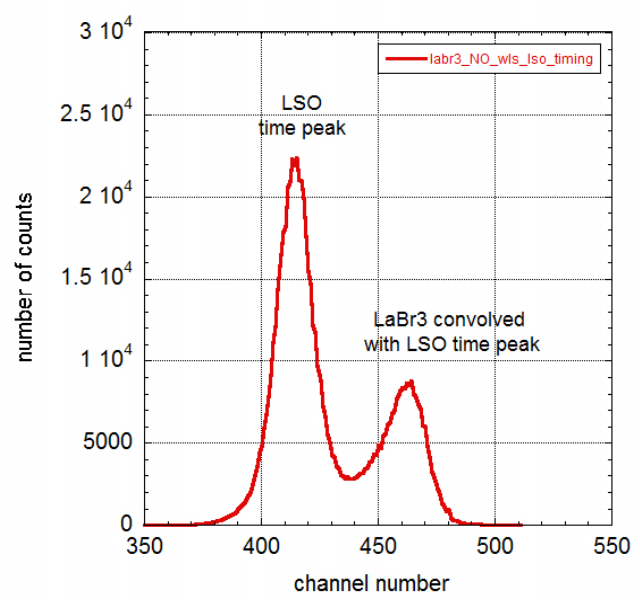

Fig 11a. PSD or timing information for a directly coupled $\mathrm{LaBr}_{3} / \mathrm{LSO}$ phoswich. The two crystals can be easily identified. 


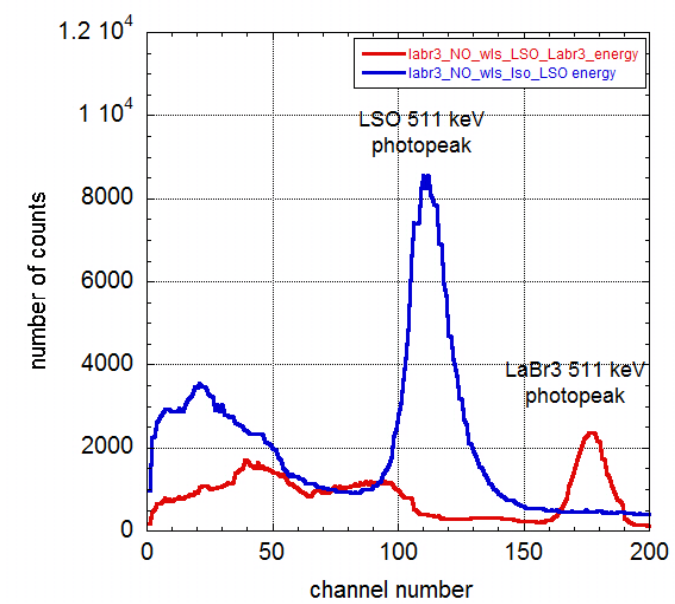

Fig 11b. Energy information for a directly coupled $\mathrm{LaBr}_{3} / \mathrm{LSO}$ phoswich. The two energy spectra can be acquired simultaneously.

\section{LIGHT OUTPUT AND COINCIDENCE TIME RESOLUTION MEAUREMENTS FOR LABR 3 WITH AND WITHOUT WLS}

In order to investigate the effect of degradation of $\mathrm{LaBr}_{3}$ light output and timing when using the WLS we measured the energy spectrum for ${ }^{137} \mathrm{Cs}$ and the coincidence resolving time for two $\mathrm{LaBr}_{3}$ crystals in coincidence with and without the WLS for the ${ }^{22} \mathrm{Na} 511 \mathrm{keV}$ photons. The light output measurement was done with a 5" PMT Hamamatsu R877. The timing set up used has been described in a recent paper by Conti et al [ 10]. The results for the light output measurement are shown in Fig 12 and the results for the timing measurements are shown in Fig 13.

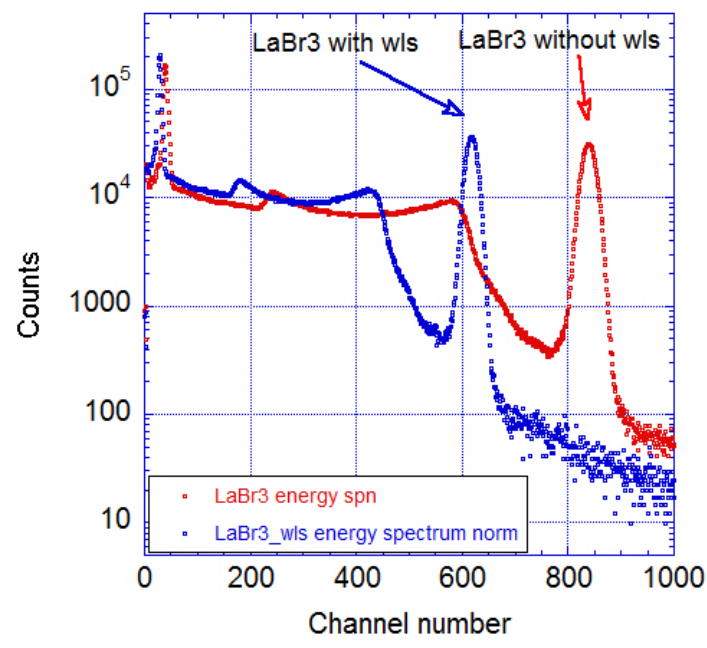

Fig 12. Energy spectra from a ${ }^{137} \mathrm{Cs}$ source for $\mathrm{LaBr}_{3}$ with and without the Eljen WLS. The ratio between the re-emitted $662 \mathrm{keV}$ photopeak and the direct $\mathrm{LaBr}_{3}$ photopeak is $\sim 0.74$ 


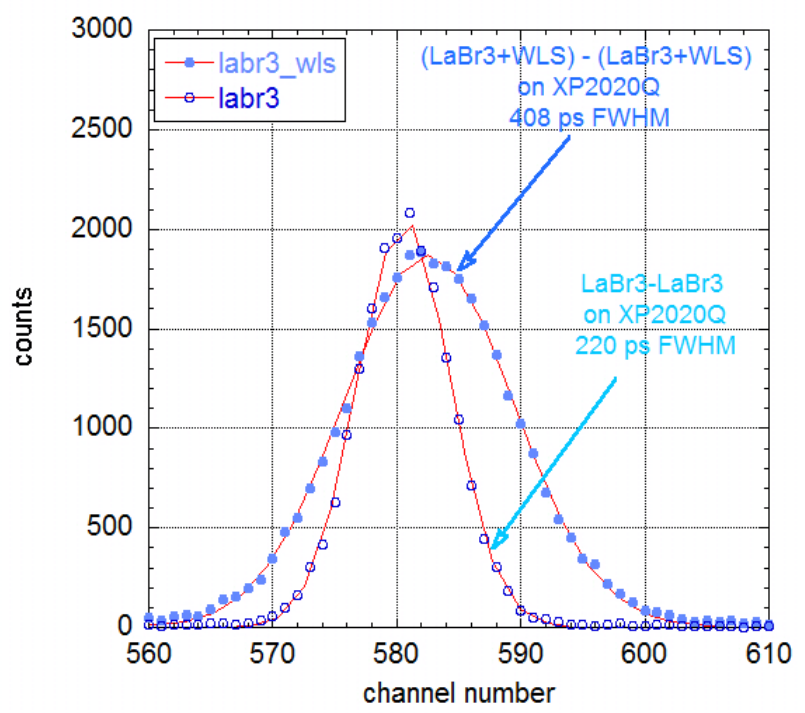

Fig 13. Coincidence time resolution results for $\mathrm{LaBr}_{3}$ with and without WLS, coupled to XP2020Q PMTs

\section{RESUlts AND Discussion}

This investigation was intended to look into the interactions between scintillators in a phoswich detector when one of the two scintillators emitted light that activated the second one. However, if the activating scintillator has a longer decay time than the activated scintillators (LSO or YSO), there would still be a convolution effect, but the scintillation time characteristics seen by the PMT would be sufficiently different to correctly identify the two phoswich components.

Originally we looked at $\mathrm{Lu}(\mathrm{Y}) \mathrm{AP} / \mathrm{LSO}$. However, the Lu(Y)AP scintillators have two to three decay components and the majority of those are much longer than the LSO decay time. This implies that the phoswich Lu(Y)AP/LSO may work well from identification point of view since the apparent Lu(Y)AP decay time is longer than LSO. Most of the problems as described in the literature come from the factor of 3-4 difference in light yield between LuYAP and LSO and how to cope with this light difference in a phoswich arrangement [9].

When using $\mathrm{LaBr}_{3}$ in a phoswich arrangement with LSO we may obtain a clearer picture of the LSO activation, since the $\mathrm{LaBr}_{3}$ used has a principal fast component of $\sim 25 \mathrm{~ns}(97 \%)$ and a small fraction of a slow component of $\sim 860 \mathrm{~ns}(3 \%)$. As Fig 5 and 6 indicate, the assumption that the resulting scintillation characteristics of the $\mathrm{LaBr}_{3}$ in a $\mathrm{LaBr}_{3} / \mathrm{YSO}$ or $\mathrm{LaBr}_{3} / \mathrm{LSO}$ phoswich, will be a convolution between $\mathrm{LaBr}_{3}$ decay and YSO(LSO) decay, seems to be verified. Our PSD data showed that the $\mathrm{LaBr}_{3}$ /YSO phoswich only gave one timing peak since the $\mathrm{LaBr}_{3}$ convolved with YSO gave time information too close to the YSO time information. By adding the WLS, a true phoswich was created and the two scintillators could now be correctly identified and the energy (and time) information from both scintillators could simultaneously be acquired. The time resolution will be degraded when using the WLS. A timing set up with $\mathrm{LaBr}_{3}$ scintillators gave 220 ns time resolution, and when placing WLS foils between the $\mathrm{LaBr}_{3}$ crystals and the PMTs the time resolution degraded to 400 ps. Obviously a LaBr 3 /WLS/LSO phoswich is not a time-of-flight (TOF) detector. The ratio between the light output from the $\mathrm{LaBr}_{3}$ with and without the WLS is 0.74 indicating that $\sim 26 \%$ light is lost in the WLS re-emission. Similarly the energy resolution degrades, going from $3.4 \%$ (662 $\mathrm{keV}$ ) to $4.1 \%$.. The WLS thickness of $0.1 \mathrm{~mm}$ had over $95 \%$ absorption in the $375 \mathrm{~nm}$ region.

We have also shown in Fig 9, that a thin YSO $(0.7 \mathrm{~mm})$ scintillator could be used as a wavelength shifter in a $\mathrm{LaBr}_{3} / \mathrm{LSO}$ phoswich and was correctly positioning the $\mathrm{LaBr}_{3}$ scintillation information in the YSO timing peak. The thin YSO crystal had $>95 \%$ absorption at $375 \mathrm{~nm}$.

Finally we have compared a $\mathrm{LaBr}_{3} / \mathrm{LSO}$ phoswich with and without WLS in Fig 10 and 11 . With the WLS the separation is good. A bit unexpected was the $\mathrm{LaBr}_{3} / \mathrm{LSO}$ data without a WLS, showing $\mathrm{LaBr}_{3}$ scintillation information appearing at a time approx $30 \mathrm{~ns}$ after the LSO peak. We believe that the difference in rise time between the LSO scintillation decay and the $\mathrm{LaBr}_{3} / \mathrm{LSO}$ convolution is the reason for this. LSO rise time is $<2 \mathrm{~ns}$ while the rise time of the convolution is $25 \mathrm{~ns}$. The set-up shown in Fig 1 is rise time sensitive.

The rise time of YSO used in the $\mathrm{LaBr}_{3} / \mathrm{YSO}$ phoswich is of the order of 10-15 ns. The rise time of the convolution between $\mathrm{LaBr}_{3}$ and YSO is $25 \mathrm{~ns}$, that is, the two decay curves are fairly close together which may explain why we only see one timing peak in the $\mathrm{LaBr}_{3}$ /YSO PSD time spectrum. 


\section{CONCLUSIONS}

This paper has looked into the phoswich concept for PET detectors and made some observations regarding scintillators that interact. A true phoswich combination has two or more scintillator components that do not interact. By using pulse shape discrimination techniques such as the one shown in Fig. 1, the two scintillators can be uniquely identified assuming different decay times. What scintillators to use and in what order to place them in the phoswich is sometimes difficult to decide The scintillator in front may be the one with the highest stopping power, but light output considerations may change this. The scintillator with the lowest light should be placed in the back, where it is close to the PMT.

Part of the investigation in this work has been the use of a wavelength shifter. A wavelength shifter may be beneficial in situations when one of the scintillators of a phoswich combination emits light into the excitation band of the other. The functionality of the wavelength shifter is then to have the same excitation/emission band characteristics as the affected scintillator. The example given in this work is the $\mathrm{LaBr}_{3} / \mathrm{LSO}$ combination. The commercial WLS from Eljen used in this paper has similar scintillation excitation and emission characteristics as LSO, with excitation around $360 \mathrm{~nm}$ and emission just above $400 \mathrm{~nm}$ but with a $3 \mathrm{~ns}$ scintillation decay time. The re-emitted light has scintillation decay time characteristics similar to $\mathrm{LaBr}_{3}$ but due to the $\mathrm{LaBr}_{3} / \mathrm{WLS}$ interaction the rise time may change to $3 \mathrm{~ns}$ if the original rise time is less than this. As mentioned in the introduction we have looked into the LuYAP/LSO phoswich. However, as mentioned in the result section, a WLS in not necessary in this case since the longer decay components of LuYAP make a regular PSD identification unique.

\section{REFERENCES}

[1] M. Schmand, L. Eriksson, M.E. Casey, M.S. Andreaco, C. Melcher, K. Wienhard, et al.,"Performance results of a new DOI detector block for high resolution PET - LSO research tomograph HRRT," IEEE Trans. Nucl. Sci., vol. 45, no. 3, 1998.

[2] V. Sossi, H.W.A.M. de Jong, W.C. Barker, P. Bloomfield, Z. Burbar, M.-L. Camborde, et al. "The Second Generation HRRT - a Multi Centre Scanner Performance Investigation", 2005 IEEE NSS Conference Records

[3] PET scanner. USPTO patent \# 7,102,135 B2 (CERN, P. Lecoq)

[4] P. Sempere Roldan, E. Chereul, O. Dietzel, et al "Raytest ClearPET, a new generation small animal PET scanner", Nucl. Instr. Meth. A 571, (2007) 498-501

[5] Annenkov A., Fedorov A., Korzhik M., et al, Industrial growth of LuYAP scintillation crystals, Nucl. Inst. Meth. A 537 (2005)182-184

[6] Musolino M, De Simons A., Morbiato A., et al, Growth and scintillation properties of large size LuYAP Crystals, presented at Scint07 conference, Wake Forest University, Winston-Salem, June 4-8, 2007

[7] Green MV., Seidel J., Xi W., Choyke PL. Light decay time/gain shift in a LaBr 3 :Ce/LYSO:Ce phoswich detector, M06-33, 2006 IEEE NSS Conference Records, San Diego

[8] L.M.Bollinger and G.E. Thomas, Rev. Sci. Instr. 32 (1961) 1044

[9] Ho Jung J, Choi Y, Hyung Chung Y, Devroede O, et al, Optimization of LSO/LuYAP phoswich detector for small animal PET, Nucl. Instr. Meth. A 571(2007)669-675.

[10] Conti M, Eriksson L, Rothfuss H, Melcher Comparing fast scintillators with TOF PET potentiality. Sorma West conference 2008 